Rev. salud pública. 10 sup (1): 109-118, 2008

\title{
El Nudo Gordiano de la Equidad de Género
}

\author{
The Gordian knot in gender equity
}

\author{
Leticia Artiles-Visbal
}

Instituto Superior de Ciencias Médicas de La Habana, Cuba. leticia@infomed.sld.cu

Recibido 2 de Marzo 2008/Enviado para Modificación 20 Julio 2008/Aceptado 13 Agosto 2008

\section{RESUMEN}

El género ha sido considerado como uno de los determinantes sociales de la salud; este hecho constituye una oportunidad para profundizar en un análisis que, por cotidiano, se convierte en invisible. La equidad de género en salud significa tener iguales oportunidades de acceso a los recursos disponibles, una distribución democrática del poder y de los conocimientos en el sistema de salud, una política de salud que beneficie a todos sin consentir privilegios debido a diferencias de sexo. El conjunto de factores que se involucran en las inequidades debidas a la construcción cultural y social asignada y asumida por mujeres y hombres constituyen un entramado complejo, un nudo gordiano. La inequidad de género se expresa en las diferentes formas en que las personas se reproducen biológica y socialmente. El género es un determinante cultural que se asume socialmente, se expresa en relaciones jerárquicas de poder y en estereotipos, desempeño de roles y, sobre todo, en el control de los recursos. El propósito del presente trabajo está dirigido a develar diferentes enlaces de este nudo gordiano, que es necesario desarticular o cortar para avanzar hacia la equidad de género en salud.

Palabras Clave: Género, desigualdades, salud, problemas sociales (fuente: DeCS, BIREME)

\section{ABSTRACT}

Gender has been considered to be one of the social determinants of health; this fact constitutes an opportunity for going deeper into an analysis which, as it is a daily matter, becomes invisible. Gender-equity in health means having equal opportunities of gaining access to the available resources, a democratic distribution of power and know-how in the health system and a health policy benefitting all without allowing privileges due to gender differences. The set of factors involved in inequities due to cultural and social construction assigned to and assumed by females and men constitutes a complex framework, a Gordian knot. Gender inequity is expressed in the different ways in which people reproduce biologically and socially. Gender is a cultural determinant which is socially assumed, expressed in hierarchical power relationships and in stereotypes, performing roles and, above all, controlling 
resources. This work is aimed at disclosing different links of this Gordian knot, which must be dismantled or cut for making advances towards gender-equity in health.

Key Words: Gender, inequality, health, social problems (source: MeSH. NLM).

\begin{abstract}
“En Gordión (actual Anatolia) había un yugo con una cuerda ceñida por un nudo complicado. Según narra la leyenda, un campesino llamado Gordias (de ahí el nombre de Gordiano) llevaba sus bueyes atados al yugo con unas cuerdas anudadas de modo imposible de desatar. Este hombre, al llegar al poblado, cumplió un augurio que promulgaba el hecho de que el futuro rey de Frigia vendría por la Puerta del Este acompañado de un cuervo que se posaría en su carro. Según las tradiciones, quien consiguiera desatar el nudo gordiano podría conquistar Oriente. Cuando Alejandro Magno 356-323 adC) se dirigía a conquistar el Imperio Persa, en el 333 AdC tras cruzar el Helesponto, conquistó Frigia en donde se enfrentó al dilema de desatar el nudo. Solucionó el problema cortando el nudo con su espada; esa noche hubo una tormenta de rayos, simbolizando según Alejandro que Zeus estaba de acuerdo con la solución, y dijo: «es lo mismo cortarlo que desatarlo». Efectivamente Alejandro conquistó Oriente" (1).
\end{abstract}

$\mathrm{E}$ l enunciado de la determinación de los factores sociales sobre la salud de las personas es de larga data y ha sido planteado y demostrado por clásicos de la salud pública, lo que ha sido resumido hábilmente por Berlinguer en su artículo Determinantes sociales de la enfermedad donde reseñaba (2): Henry E. Sigerist expresó que «en cualquier sociedad dada, la incidencia de la enfermedad estaba determinada mayormente por factores económicos y que una baja calidad de vida, falta de comida, ropa y combustible, malas condiciones de vivienda y otros síntomas de pobreza han sido siempre las mayores causas de enfermedad»; Virchow señaló que "si la medicina deseaba lograr sus objetivos por completo, debía entrar por completo a la vida política, y debía indicar todos los obstáculos que impedían la consumación normal del ciclo de vida", por su parte Berlinguer enfatizó en su artículo (2): “...la idea central es que la medicina y los servicios de salud constituyen sólo uno de los factores que tiene influencia sobre la salud de la población. En realidad, los factores principales se hallan en el amplio espectro de condiciones sociales y económicas en que vive la gente: la pobreza en sus diversas manifestaciones, las injusticias, el déficit de educación, la inseguridad en la nutrición, la marginalización social y la discriminación, la protección insuficiente de la infancia temprana, la discriminación contra la mujer, la vivienda insalubre, el deterioro urbano, la falta de agua potable, la violencia generalizada, las brechas y disparidad en los sistemas de seguro social”. Estas breves citas de clásicos de la Salud Pública que llegan lamentablemente "vivas" hasta nuestros días, nos colocan ante una verdad «supuestamente de perogrullo», la desigualdad social genera enfermedad, sin embargo en pleno siglo XXI continua como un problema no resuelto. 
Esta breve reseña nos coloca un acertijo: si es bien conocido que las desigualdades injustas constituyen un núcleo determinante de la salud de las personas, si la salud de las personas son una base fundamental del desarrollo de las fuerzas productivas, y estas a su vez del desarrollo, si se reconoce que la condición de clase social, de territorio, de color de la piel, de etnia, de diversidad sexual y de sexo, coloca a las personas en condiciones de deterioro individual y social, y provocan enfermedad y muerte, ¿por qué no han tenido una respuesta?; ¿por qué no se ha dado solución a las situaciones sociales que "fagocitan" personas y poblaciones? Estamos en presencia de un nudo gordiano, con agravantes sustantivos condicionados por la depredación del medio ambiente, por el agotamiento de las fuerzas de energía y del agua, por la cultura consumista, por el enriquecimiento progresivo de unos pocos que se apropian de las riquezas de todos, por una cultura guerrerista y excluyente de los poderosos diseminando enfermedades y muertes. En estas condiciones, resulta perentorio cortar este nudo para tomar el cielo por asalto, para lo que resulta necesario la unión de todas las fuerzas progresistas en un frente unido para lograr ese mundo posible al que todos y todas aspiramos. El propósito del presente trabajo está dirigido a develar diferentes enlaces del nudo gordiano que son necesarios desarticular o cortar para avanzar hacia la equidad de género en salud.

El nudo de la inequidad de género

"La equidad en salud significa tener iguales oportunidades de acceso a los recursos disponibles, una distribución democrática del poder y de los conocimientos en el sistema de salud, una política de salud que beneficie a todos sin consentir privilegios debido a diferencias de raza, género, territorio, discapacidad u otro rasgo de distintividad grupal o personal” (3).

El género es una construcción simbólica que reside en identificar las expectativas y valores que una cultura concreta asocia al hecho de ser mujer u hombre, al carácter y la calidad de las relaciones que se establecen entre los mismos. Esta categoría se define como el "conjunto de asignaciones culturales que diferencia a los hombres de las mujeres, los articula dentro de relaciones de poder frente a los recursos y se expresa en símbolos, estereotipos, desempeño de roles y en el modo de actuación social en los espacios donde se genera la continuidad cultural” (4). Al introducir el género como criterio de análisis, consideramos como punto de partida que la diferencia de género se traduce en múltiples formas de desigualdad y ausencia de equidad en las relaciones entre los sexos y, como punto de llegada, el necesario alcance de la equidad e igualdad social entre los sexos como elemento sustantivo para lograr la calidad de vida de las poblaciones. 
Para expresar las inequidades de género basta analizar algunas cifras actuales, reportadas en el Informe sobre Desarrollo Humano del 2006 (5). En las Tablas 1 y 2, se presentan un pequeño número de países que contrasta desde el país que ocupa la posición $n^{\circ} 1$ del IDH, Noruega, con Níger, que ocupa la última posición $\mathrm{N}^{\circ} 177$.

En el reporte del Programa de las Naciones Unidas para el Desarrollo (PNUD) se pueden observar algunos enlaces del nudo que muestran la inequidad de género.

Primer enlace del nudo: la Esperanza de Vida al Nacer

La expectativa de vida en Noruega es de 79,6 años mientras que en Níger es solo de 44,6; el valor diferencial entre uno y otro es de 35 años. Dentro de nuestra América, la diferencia de Bolivia respecto a Níger sólo es de 19,8 años. Si analizamos la relación de feminidad (tasa femenina/masculina) podremos observar (Tabla 2), que las mujeres tienen en general una esperanza de vida mayor, excepto para Níger que es igual a 1,00. Es conocida la supervivencia femenina lo que algunos atribuyen a una ventaja adaptativa vinculada al cromosoma X para garantizar la reproducción de la especie. Por tanto, si las mujeres están muriendo más de lo que deben morir, este diferencial se debe esencialmente no a factores biológicos sino a factores sociales. Obsérvese que el Índice de Esperanza de Vida en Noruega es de 0,91 mientras que en Bolivia es de 0,66 y en Níger 0,33.

El hecho de que la esperanza de vida de las mujeres sea mayor, ha tendido a opacar la realidad de su calidad de vida. En este sentido, se puede afirmar que las mujeres no viven más, sino que mueren más tarde. En condiciones de pobreza, esta "supuesta" ventaja disminuye considerablemente; es decir, las más pobres mueren más de lo que debieran morir; las cifras son elocuentes.

Segundo enlace del nudo: los dineros

En la Tabla 1 se puede observar que el PIB per cápita para el año 2004 fue de 32 454 para Noruega y de 779 para Níger, para Bolivia 2720 lo que se traduce en una diferencia respecto a Níger de 37675 para Noruega y sólo de 1941 para Bolivia. Pero si consideramos la relación mujer/hombre respecto a los ingresos medios, la supremacía masculina es muy superior a la femenina en todos los países, marcadamente diferencial en la medida que los países se ubican en un IDH más bajo. Si se articula ello con la marginación de las mujeres de la propiedad 
de la tierra, del acceso al crédito, de la educación, de la toma de decisiones, la imposibilidad de acceso a la planificación familiar, el sometimiento en que viven la sexualidad, la violencia doméstica, las jornadas de trabajo interminables, la desigualdad de género supone uno de los principales obstáculos que impide a las mujeres participar y disfrutar de los beneficios del desarrollo con plenitud.

Tercer enlace del nudo: la educación

En la Tabla 2 se observa que en general la tasa de matriculación en la enseñanza primaria, secundaria y terciaria es mayor ligeramente en las mujeres que en los hombres, excepto para Bolivia y Níger, donde es inferior. Esto indica que, en la medida en que los países tienen menor desarrollo, son más pobres y las mujeres están por debajo en el proceso educativo. Esta brecha se traduce en menor acceso a la información, menor capacidad para el autocuidado y cuidado de la prole, inferior competencia para acceso a trabajos remunerados y acceso a posibilidades de crédito y autodesarrollo.

Tabla 1. Indicadores de desarrollo para países seleccionados

\begin{tabular}{|c|c|c|c|c|c|c|c|c|}
\hline & \multirow[t]{2}{*}{$\begin{array}{l}\text { Pasioion } \\
\text { IDH }\end{array}$} & \multirow[t]{2}{*}{$\begin{array}{l}\text { Valor } \\
\text { IDH }\end{array}$} & \multicolumn{2}{|c|}{ EVN (arios) } & \multicolumn{2}{|c|}{$\begin{array}{c}\text { PIB Fer cap:a } 2004 \\
\text { (USD) }\end{array}$} & \multirow{2}{*}{$\begin{array}{l}\text { Indioe } \\
\text { Esperanzs } \\
\text { Vida }\end{array}$} & \multirow[t]{2}{*}{$\begin{array}{l}\text { Indce } \\
\text { Educación }\end{array}$} \\
\hline & & & Valor & $\begin{array}{l}\text { Diferencial } \\
\text { outilimo }\end{array}$ & & $\begin{array}{l}\text { Diferencisl } \\
\text { cultimo }\end{array}$ & & \\
\hline Nonvege & 1 & 0,965 & 79,6 & 35 & 38454 & 37.675 & 0,91 & 0,99 \\
\hline USA & 8 & 0,948 & 77,5 & 32,9 & 39876 & 38.097 & 0,80 & 0,97 \\
\hline Cubs & 50 & 0,826 & 77.6 & 33 & - & & 0,88 & 0.93 \\
\hline México & 53 & 0,021 & 75,3 & 30,7 & 9.000 & 9.024 & 0,84 & 0,06 \\
\hline Celembia & 70 & 0,790 & 72,6 & 28 & 7256 & 6.477 & 0,79 & 0,86 \\
\hline Venezuela & 72 & 0.784 & 73 & 28,4 & 6.043 & 5.264 & 0.8 & 0.87 \\
\hline Bolvia & 115 & 0,692 & 64,4 & 19.8 & 2720 & 1,941 & 0,65 & 0,87 \\
\hline Niger & 177 & 0,311 & 44.8 & & 779 & & 0,33 & 0.26 \\
\hline
\end{tabular}

Cuarto enlace del nudo: posición de la mujer

El Índice de Potenciación de Género es un indicador utilizado por el Informe de Desarrollo Humano (5) para indicar el posicionamiento de la mujer; se evalúa por el porcentaje de escaños parlamentarios ocupados por mujeres. En la Tabla 2 se indica que en Noruega existe un 37,9 \% de escaños ocupados por mujeres; en Cuba, a pesar de ser un país en vías de desarrollo, 36 \% parlamentarias, lo que se corresponde a una política de desarrollo participativo de la mujer en todas las esferas de gobierno; Níger solo tiene ocupado un 5,8 $\%$ de escaños. La variabilidad entre países es grande. Este Indicador a nuestro juicio resulta insuficiente, pues el porcentaje de participación no es un indicador directo de la capacidad de decisión; en condiciones de una prevalencia 
de escaños masculinos, las mujeres acceden, participan, proponen normativas y leyes, pero en la toma de decisiones todavía el voto masculino puede limitar la verdadera fuerza femenina en esos escenarios; indiscutiblemente las mujeres constituyen una fuerza pujante, contestataria, pero no pocas veces devienen en "grupos silenciados" que limitan el real alcance que pueden tener sus planteamientos.

Otros enlaces del nudo involucrados en la inequidad de género

$\mathrm{Al}$ analizar el ámbito de la salud, es necesario enfatizar en las mujeres, porque mujeres y hombres ocupan posiciones diferentes frente a la utilización y la provisión de atención; es necesario enderezar la balanza entre necesidades y recursos y entre contribuciones y beneficios. Las mujeres, respecto de los hombres, presentan mayor incidencia de trastornos agudos a lo largo de la vida, mayor prevalencia de enfermedades crónicas no mortales y niveles mas elevados de discapacidad en el corto y largo plazo, particularmente dentro de las edades avanzadas. Esta diferencia se mantiene entre los países y en diferentes estratos sociales. La morbilidad femenina unida a los requerimientos exclusivos de la función materna, hacen que la necesidad de servicios de atención exceda a la de los hombres. El reconocimiento de la desventaja generalizada de las mujeres en cuanto al acceso a recursos está en la base de los argumentos de las agencias internacionales y los gobiernos para focalizar la población femenina, lo que ya fue planteado en el primer Informe de Desarrollo Humano, donde se declaró: "ningún país del mundo trata tan bien a sus mujeres como a sus hombres" (6)

Tabla 2. Índice de desarrollo de género

\begin{tabular}{|c|c|c|c|c|c|c|c|c|c|c|c|}
\hline \multirow[t]{2}{*}{$\begin{array}{l}\text { IDG } \\
\text { Rango }\end{array}$} & \multirow[t]{2}{*}{$\begin{array}{l}\text { IDG } \\
\text { Vator }\end{array}$} & \multicolumn{3}{|c|}{$\begin{array}{l}\text { ESPERANZA VIDA } \\
\text { NACER }\end{array}$} & \multicolumn{3}{|c|}{$\begin{array}{l}\text { Tirsa truta matricats } \\
\text { primaria, secundaria y } \\
\text { serciaria combinada (\$) }\end{array}$} & \multicolumn{3}{|c|}{$\begin{array}{l}\text { Irgrescos madios } \\
\text { estmados (PPA en USD }\end{array}$} & \multirow{2}{*}{$\begin{array}{c}\text { Eacaficas } \\
\text { Parlamentarice } \\
\text { por mujeres ( } \\
\text { pob total) }\end{array}$} \\
\hline & & Mujer & Hombre & $\mathrm{HMM}$ & Muler & Hombre & HM & Muper & Homtre & нм & \\
\hline 1 & 0,98 & 82 & 77,1 & 1,063 & 106 & 98 & 1,093 & 33,0 & 43.9 & 0,751 & 37,9 \\
\hline 8 & 0,94 & 80,2 & 74,8 & 1,072 & 97 & 69 & 1,009 & 30,5 & 490 & 0,623 & 15 \\
\hline 0 & 0 & 79,5 & 76.8 & 1.035 & 81 & 78 & 1,028 & 0 & 0 & 0 & 36 \\
\hline 15 & 0,81 & 77,8 & 72,8 & 1,068 & 76 & 75 & 1,013 & 5,5 & 142 & 0,398 & 25 \\
\hline 58 & 0,78 & 75,8 & 69.8 & 1,088 & 74 & 71 & 1,042 & 5,3 & 22 & 0,582 & 10,8 \\
\hline 60 & 0,78 & 76,1 & 70,2 & 1,084 & 76 & 73 & 1,041 & 4,0 & 79 & 0,511 & 18 \\
\hline 86 & 0,68 & 66,5 & 62,3 & 1,067 & 83 & 89 & 0,932 & 1,9 & 3,4 & 0,572 & 14,6 \\
\hline 120 & 0,44 & 43,5 & 432 & 1,005 & 50 & 60 & 0,033 & 669 & 1.6 & 0,410 & 5,0 \\
\hline
\end{tabular}

Fuente: PNUD. Índice de Desarrollo de Género (IDG), 2006

No hay que pensar mucho para evidenciar que el condicionamiento de género determina diferentes formas de vivir, enfermar y morir por causas prevenibles; configura un perfil epidemiológico específico, de género, en 
función de que cada sexo, tiene características y necesidades diferentes, y requiere de respuestas correspondientes; la base de esta configuración cultural se articula directamente con el control que exista sobre los recursos según su diferencial específico de territorio, clase social, color de la piel y etnia, lo que se manifiesta en cifras inobjetables (7) :

- La carga de enfermedad de salud sexual y reproductiva es del $22 \%$ para las mujeres mientras que para los varones es del $3 \%$, diferencias que podrían estar vinculadas con cuestiones relativas a sus prácticas de sexualidad y otros aspectos socioculturales.

- La salud materna es la carga dominante en la enfermedad por salud sexual y reproductiva de mujeres y en Latinoamérica y el Caribe (LAC) representa $11,3 \%$ de la carga total de enfermedad.

- La carga de enfermedad de la salud sexual y reproductiva no se limita a los efectos sobre la mujer misma, sino que tiene a su vez efectos intergeneracionales: las causas perinatales son el $10 \%$ del total de DALY's, (Disability Adjusted Life Years) perdidos asociados a enfermedad reproductiva.

- El $12 \%$ de las muertes de las mujeres en edad reproductiva y el $15 \%$ de los DALY's perdidos son resultado del sexo no seguro. La mayoría de las consecuencias del sexo no seguro recaen sobre las mujeres, quienes sufren de enfermedad pélvica inflamatoria, cáncer cervicouterino, infertilidad y complicaciones de abortos inseguros. En hombres, la carga de la enfermedad por sexo no seguro es también alta debido a las altas proporciones de casos de SIDA que se registran.

- El acceso a un aborto seguro es posible en Puerto Rico, Guyana y Cuba; en tanto que en Chile, El Salvador y Honduras está prohibido. En el resto de los países de LAC se acepta en determinadas circunstancias como la violación, el peligro para la salud y la probabilidad de muerte de la mujer, las malformaciones del feto, por causas accidentales o imprudenciales, debido a inseminación no consentida o por razones económicas (por ejemplo, en Yucatán, México).

- Las estadísticas de mortalidad materna y de aborto están íntimamente ligadas a situaciones de violencia. 


\section{Consideraciones finales para cortar el nudo}

Las inequidades de género en salud persistentes evidencian que es prácticamente imposible desenredar el nudo. Se trata de cortarlo y esto requiere de una perentoria respuesta. Está demostrado en la práctica que esta exigencia va más allá de las buenas intenciones declaradas en las Conferencias de Población celebrada en El Cairo (1994) y la Conferencia de la Mujer Celebrada en Beijing (1995), y más recientemente en la Declaración de los Objetivos del Milenio (8-10). A pesar de las declaraciones formales, de la adscripción de los países a supuestos mandatos vinculantes internacionales, las mujeres siguen privadas de préstamos, de herencia y no se reconoce su trabajo. Sus necesidades en materia de atención en salud y nutrición no son prioritarias, carecen de acceso adecuado a la educación y a los servicios de apoyo, y su participación en la adopción de decisiones en el hogar y en la comunidad es mínima. Atrapada en el ciclo de la pobreza, la mujer carece de acceso a los recursos y a los servicios para cambiar su situación. La brecha que separa a los hombres de las mujeres en el ciclo de la pobreza se sigue ampliando, de allí que se continúe enfatizando en la feminización de la pobreza.

Es necesario desarrollar las fuerzas de la sociedad civil, empoderar a la sociedad, mujeres y hombres, para que adquieran verdadera conciencia de derechos; para que la posición de las mujeres en su acceso al poder, sea real y no formal, y que en su participación en las políticas públicas dejen de "constituir grupos silenciados” ante el indiscutible dominio patriarcal.

Para ello, es necesario formar un frente único, trazar estrategias y traducirlas en acciones prácticas para enfrentar al modelo de desarrollo social y económico prevalerte en la Región de Las Américas, profundizado por el conjunto de políticas neoliberales en el marco del proceso de globalización, sustentado en una lógica de mercado, de privatización y medicalización de la salud, en detrimento del disfrute de la salud como bien público y derecho ciudadano. La acción debe basarse en la concientización de que la violencia en general y, en particular, la violencia basada en el género, constituye un problema de salud pública, y no un problema privado. Todas las instituciones jurídicas, policiales, educativas, de salud y todas las que vinculan directamente o indirectamente con esta triste epidemia universal deben dar respuesta real, efectiva y no formal. Ser conscientes de que, como señala la Carta de Brasilia, documento que se concertó en la Reunión Regional de consulta con la sociedad civil sobre los determinantes sociales de la salud, en Brasil, en el 2007: "todo el modelo neoliberal castrante agudiza las violaciones a los 
derechos humanos, y las desigualdades que llevan a las inequidades sanitarias que fragilizan y afectan las situaciones de salud y de vida que son completamente evitables e injustas".

Todavía falta un trecho a las mujeres para lograr la equidad social y en salud. Esto significa un reto, que se traduce en la necesidad de incrementar las capacidades personales, empoderar a las mujeres en el reconocimiento conciente de sus valores y fuerzas, no sólo reproductoras biológicas, sino de reproductoras sociales.

ALAMES, en la Declaración de Bahía (11), declaró que las inequidades sociales tienen como verdadera raíz la división de clases sociales, lo que para nuestro objeto de estudio significa la agudización de las inequidades de género en salud. Es necesario fortalecer la articulación entre la academia y la fuerza de los movimientos sociales, que permitan un avance conjunto y sinérgico entre teoría y práctica, ciencia y política, como una de las estrategias para la defensa de la salud como bien público y derecho ciudadano. La Coordinación General de ALAMES y, en particular, la Red de Género y Salud Colectiva, tiene el compromiso de desarrollar acciones para que la Academia salga de su torre de marfil y se articule con los movimientos sociales, de forma bidireccional, agregando valor para unos y otros, instalando capacidades, accediendo a los medios de comunicación, desarrollando herramientas prácticas que ponga en manos de las organizaciones de la sociedad civil, en especial, de las mujeres, para su defensa e interfase de acceso a todos los niveles, agregando valor a los procesos que desarrollen de manera conjunta. Estas acciones pueden contribuir significativamente a construir la espada que corte el nudo gordiano

\section{REFERENCIAS}

1. Definición de nudo gordiano. Enciclopedia Wikipedia. [Internet] Disponible en http://es.wikipedia.org/ wiki/Nudo_gordiano». Consultado en 8/10/2007.

2. Berlinguer G. Determinantes sociales de las enfermedades. Rev Cubana Salud Pública[online]. 2007,33(1) [ïnternet]. Disponible en: <http://scielo.sld.cu/scielo.php?script=sci_arttext\&pid=S086434662007000100003\&lng=es\&nrm=iso>. Consultado en 12/08/08

3. Torre ME. Salud para todos sí es posible. $1^{a}$ Ed. La Habana: Sociedad Cubana de Salud Pública. Sección de Medicina Social. La Habana; 2005. p.200

4. Artiles L. Importancia de la conciencia de género para las políticas públicas en salud y los derechos ciudadanos. Revista de Ciencias Sociales Universidad de Costa Rica. 97 (III). 2002; pp.127135

5. Programa Naciones Unidas para el Desarrollo, PNUD. Informe sobre Desarrollo Humano 2006. Poder, pobreza y la crisis mundial del agua Programa de las Naciones Unidas para el Desarrollo. New York; 2006. pp.363-370

6. Programa Naciones Unidas para el Desarrollo, PNUD, Human Development Report 1995, New York, PNUD; 1995. p.86 
7. Fondo de Población de las Naciones Unidas, UNFPA. Equipo de Apoyo técnico para América Latina y el Caribe. Pobreza, salud sexual y reproductiva y derechos humanos. UNFPA, México DF; 2005. p.235

8. Fondo de Población de las Naciones Unidas, UNFPA. Informe. Conferencia Internacional sobre la Población y el Desarrollo, El Cairo, Egipto, septiembre de 1994. UNFPA, Venezuela; 2003. pp.19-25

9. Naciones Unidas. Cuarta Conferencia Mundial sobre la Mujer. A/Conf. 177/20; sept 4-15; Naciones Unidas, Beijing; 1995. pp.45-136

10. Naciones Unidas. Declaración del Milenio. Resolución 55,2 Asamblea General [Internet]. Disponible en: http://www.un.org/spanish/millennium goals/ares552.html.2000. Consultado en 10/10/2007

11. Declaración de Salvador de Bahía. X Congreso de ALAMES, IV Congreso Brasileño de Ciencias Sociales y Humanas en Salud, Congreso XIV de IAHP y X de ALAMES. 18 de julio del 2007. [Ïnternet]. Disponible en http://www.idisa.org.br/site/download/DECLARACION DESALVADORBAHIA2007.doc. Consultado en 9/10/2007. 